\title{
El modelamiento matemático como vía idónea para la formación de ingenieros. Una reflexión pedagógica
}

\author{
The Mathematical Modeling As Ideal Way To The Formation Of Engineers. A \\ Pedagogical Reflection
}

\section{A Modelagem Matemática Como Ideal lugar à formação de engenheiros. Uma Reflexão Pedagógica}

\author{
John J. Leal ${ }^{1}$ \\ Juan P. Cardona² \\ Alexánder Agudelo ${ }^{3}$
}

Fecha de recepción: junio 2014

Fecha de aceptación: noviembre 2014

Para citar este artículo: Leal, J. Cardona, J. y Agudelo, A. (2015). El modelamiento matemático como vía idónea para la formación de ingenieros. Una reflexión pedagógica. Revista Científica, 21, 91-96. Doi: 10.14483/udistrital.jour.RC.2015.21.a9

\section{Resumen}

El quehacer de los ingenieros consiste en modelar situaciones particulares en campos específicos de su saber, definir sistemas y variables de interés, diseñar o utilizar modelos matemáticos que relacionen las variables seleccionadas como relevantes, estudiar distintas alternativas para la solución de modelos bien se analítica o numéricamente, definir márgenes de error para poder comparar tales soluciones con las obtenidas por sistemas computacionales especializados diseñados para tal fin y comparar las soluciones con resultados experimentales y reformular los modelos para minimizar sus errores. En el proyecto "Diseño y evaluación de una estrategia didáctica para resolver problemas de ingeniería utilizando modelamiento matemático y ecuaciones diferenciales ordinarias", los estudiantes de ingeniería de la Universidad Cooperativa de Colombia, formularon y resolvieron modelos matemáticos para situaciones particulares, con los cuales se aproximaron al ejercicio real de su práctica profesional.

Palabras Clave: enseñanza en ingeniería, modelamiento matemático, estrategia didáctica.

\begin{abstract}
The work of engineering is to model particular situations in specific areas of their knowledge, the engineer define systems and variables, designs or use mathematical models that relate the variables as relevant, they study different alternatives to solve the models either analytically or numerically, predefine margins of error to compare these solutions with those obtained by specialized computer systems designed for this purpose, finally they compared the solutions with experimental results and reformulate their models to reduce their mistakes. In the project
\end{abstract}


“Design and evaluation of a teaching strategy for engineering problem solving using mathematical modeling and ordinary differential equations", engineering students of the Universidad Cooperativa de Colombia formulated and solved mathematical models for everyday situations approaching the exercise of their professional practice.

Keywords: Teaching in engineering, Mathematical modeling, Teaching strategy.

\section{Resumo}

O trabalho dos engenheiros é modelar situações particulares em áreas específicas de seus conhecimentos, sistemas e definir variáveis de interesse, desenhar ou utilizar modelos matemáticos que relacionam as variáveis selecionadas como relevantes, explorando diversas alternativas para a solução de modelos analíticos são numericamente ou, as margens de erro predefinidos para comparar estas soluções com os obtidos por sistemas de computador especializados destinados a esse fim, finalmente, comparando as soluções com os resultados experimentais e reformular modelos para minimizar os erros. No "Projeto e avaliação de uma estratégia educativa para resolver problemas de engenharia utilizando modelagem matemática e equações diferenciais ordinárias", projeto estudantes de engenharia da Universidade Cooperativa da Colômbia, formulados e resolvidos modelos matemáticos para situações particulares aproximar o exercício efectivo do seu prática profissional.

Palavras-chave: Engenharia de Ensino, Modelagem Matemática, estratégia de ensino

\section{Introducción}

En el medio educativo es frecuente la queja de los docentes, en todos los niveles del sistema, sobre la deficiente capacidad de apropiación de conocimientos y del escaso dominio conceptual con que llegan los estudiantes a cursar asignaturas o temas propios de determinado programa curricular; situación que se acentúa con mayor fuerza en el campo de la ingeniería, toda vez que allí el conocimiento cobra sentido en la aplicación orientada a resolver problemas o situaciones de la vida cotidiana, más allá de la elaboración abstracta desarrollada por cada disciplina.

Lo anterior indica que el estudio de la ingeniería no busca una formación propedéutica, más bien se trata de orientar a los estudiantes para que alcancen un grado de apropiación de conocimientos específicos que les permita resolver problemas con la solvencia e idoneidad que exige este campo, lo que significa adquirir la experiencia que su rol profesional demanda; ante este desafío, la decisión pedagógica es decisiva, pues es preciso optar por una selección de contenidos de enseñanza (el qué), así como proponer una forma de transmisión o estrategia didáctica (el cómo).

A propósito de qué enseñar, en el campo de las ciencias es una decisión establecida de un modo relativamente estable, mientras que en ingeniería puede ser contingente, móvil, cambiante; el contenido se trasforma al ritmo que se complejizan los estilos de vida y en la medida en que la dependencia de la tecnología se acrecienta. Así las cosas, la decisión sobre cómo enseñar tampoco puede ser ajena a tal exigencia, pues los ingenieros se ven abocados a incorporar los cambios mencionados y a adaptarse al ritmo de dichas transformaciones. Esto no quiere decir que la selección de contenidos tenga que ser "a la carta", o de acuerdo con requerimientos particulares, sino que es preciso valorar su pertinencia y su adaptación a las nuevas circunstancias (no se trata de proporcionar un "nuevo" conocimiento para cada problema, pues el avance de las ciencias no solo ha sido vertiginoso, sino que se ha adelantado a su propia época, y ahora es esta la que debe alcanzarlo).

Mientras en el progreso de las ciencias la instauración de un paradigma (Kuhn, 2004) o, dicho de otro modo, el establecimiento de una teoría se logra en el momento en que es aceptada como verosímil, en virtud de dar respuesta a problemas existentes o cuestionamientos propuestos por la propia disciplina, el nivel de generalización teórico (creación de soluciones universales) que permite 
satisfacer problemas tipo obedece, en principio, a una situación ideal, hipotética, que se postula como derrotero que orienta la investigación.

El asunto pedagógico en ingeniería reside en que, en contexto de práctica profesional, al conocimiento no se le pide que sea simplemente verosímil, ya que esta condición se puede expresar por medio de argumentaciones lógicas y con demostraciones que sean consistentes teóricamente; al ingeniero se le pide que su saber se materialice, que su efecto impacte sobre las condiciones de existencia de la sociedad y facilite la asimilación de sus propias transformaciones.

El desafío que se presenta en la enseñanza para el campo de la ingeniería consiste, entonces, en lograr transmitir un conocimiento junto con las estrategias, técnicas o métodos que lo hagan susceptible de ser convertido en tecnología, es decir, en conocimiento aplicado. La cuestión no es simple, pues desde la teoría pedagógica este asunto al parecer no ha sido abordado. Veamos dos ejemplos:

Desde la teoría del discurso pedagógico (Bernstein y Díaz, 1985) surge una paradoja por cuanto el escenario educativo es tomado como un dispositivo de transmisión-reproducción del discurso pedagógico, que se nutre de un saber que proviene del campo de la producción teórica (contexto científico) y que se transmite y legitima a través de la enseñanza y la evaluación en la institución educativa (se recontextualiza).

La paradoja consiste en que el contenido a enseñar hace parte del discurso pedagógico reproducido o recontextualizado en el escenario educativo (Bernstein ), pero bajo la idea de que la resolución de problemas específicos implica aprender a "hacer con el saber", lo que conduce a la creación de un nuevo conocimiento aplicado, por lo que tal conocimiento no siempre es transmisible, por lo menos en el contexto del dispositivo educativo en el campo de la ingeniería.

De otra parte, para la teoría de la transposición didáctica (Chevallard, 1998), el saber proveniente de un contexto científico, donde es producido, sufre cierto "tratamiento" por parte del docente quien lo transforma, adaptándolo a las condiciones del contexto de enseñanza para hacerlo accesible o comprensible a los estudiantes, en suma, lo didactiza. Esta decisión de adecuar el saber a objetivos de aprendizaje al nivel educativo que se trate implica que siempre habrá una pérdida en el nivel de profundidad, de fundamentación o de abstracción; para el caso de la ingeniería, dicha pérdida no puede ser muy considerable porque haría inútil el saber, es decir, en tanto se trivialice el saber, ya no sería aplicable.

En razón a las anteriores consideraciones, creemos que la propuesta de confrontación de ejercicios de modelamiento matemático con prácticas de laboratorio podría constituirse en un tipo ideal para apropiar conocimiento en ingeniería, esto es, para resolver problemas a partir de la puesta a prueba de un dominio conceptual que evidencie la experiencia del ingeniero.

\section{El Contexto de la Ingeniería}

\section{El quehacer del Ingeniero Consiste en Modelar Matemáticamente}

El ingeniero utiliza su experiencia para abordar una situación "real" y ubicarla en un campo de conocimiento específico con el cual se analizará dicha situación, seguidamente define un sistema (su frontera, su interior, variables internas o de estado, variables exógenas que casi siempre se aproximan a valores constantes), sus variables de entrada-salida y considera las posibles formulaciones de modelos matemáticos acordes con la situación; dependiendo de las relaciones entre las variables involucradas, selecciona los más pertinentes (Hritonenko y Yatsenko, 2003).

A partir de las relaciones matemáticas entre las variables involucradas en el proceso (entrada-salida), es necesario hacer simplificaciones que permitan resolver el modelo y predecir el comportamiento del sistema; dicho de otro modo, a una entrada particular le debe corresponder una salida determinada. Se dirá que un modelo matemático 
(v. g., ecuaciones diferenciales ordinarias o parciales) tiene solución cuando existe una función que satisface el modelo, la cual se puede encontrar analíticamente, de manera aproximativa o numérica, también es posible resolverlo utilizando simulaciones con Octave, Wxmaxima, Matlab u otras.

El ingeniero valida la solución obtenida utilizando software especializado (QuickField, Ansys, Comsol, Algor) que generan soluciones similares a las obtenidas previamente, o contrastando la solución con datos experimentales. Posteriormente se analiza o se realiza una comparación entre los distintos resultados y se define un margen de error permisible para la solución. De encontrarse que el modelo satisface la condición, se acepta y se utiliza en general para hacer predicciones de otros casos particulares bajo condiciones similares. Si la solución no cumple con el estándar definido (margen de error), es necesario revisar el proceso completo del modelamiento matemático.

En caso de no tener la teoría correspondiente, el ingeniero construye una nueva basada en las matemáticas.

\section{Modelamiento Matemático en el Proceso de Formación de Ingenieros}

Conscientes del rol que juega el modelamiento en la ingeniería, pretendemos recrear el proceso de modelamiento matemático descrito anteriormente en un contexto de enseñanza, desarrollado en el marco del proyecto de investigación: "Diseño y evaluación de una estrategia didáctica para resolver problemas de ingeniería utilizando modelamiento matemático y ecuaciones diferenciales", que contó con la financiación de la Universidad Cooperativa de Colombia. Con este proyecto se busca que los estudiantes diseñen o utilicen modelos matemáticos para comprender y resolver problemas particulares.

La pregunta de cómo "aplicar" los conceptos de matemáticas a situaciones cotidianas o de ingeniería es un asunto del cual nos ocupamos en primer lugar; simultáneamente con la presentación de los contenidos matemáticos específicos —para nuestro caso ecuaciones diferenciales ordinarias o parciales-, los cuales se muestran en contextos puntuales específicos, se definen sistemas particulares, se presentan las variables que intervienen (entrada-salida), y se formulan modelos utilizando ecuaciones diferenciales ordinarias o parciales tal y como lo haría un ingeniero experto. Al igual que en el ejercicio profesional de la ingeniería, en nuestro contexto de enseñanza también se realizan simplificaciones, debido a que en principio es necesario comprender la situación más simple posible, es decir, teniendo en cuenta el mínimo número de variables y de relaciones matemáticas entre dichas variables, para posteriormente complejizar los análisis incluyendo más variables y relaciones.

Puesto que los estudiantes con los cuales estamos trabajando se encuentran en proceso de fundamentar las técnicas básicas para la formulación y solución de modelos relacionados con ecuaciones diferenciales, es preciso hacer el proceso simultáneo de la presentación de los conceptos básicos y de las maneras de formular y plantear modelos matemáticos, así como las distintas soluciones posibles de estos. Se propone entonces a los estudiantes que seleccionen una "situación" particular de ingeniería o de la vida cotidiana, la cual se estudia durante el semestre; apoyados en la mirada del docente experto tanto en las técnicas teóricas como en la formulación y solución de modelos matemáticos se orienta un proceso que permite a los estudiantes aproximarse al ejercicio real de ingeniería.

Para resolver las ecuaciones formuladas se procede de forma analítica para luego corroborar las soluciones utilizando software como Wxmaxima, Mathematica (Wolfram, y Beck, 1994), Maple (Richards, 2002), entre otros. De no ser posible hacer esto debido a que las técnicas analíticas no lo permiten, se utilizan los métodos numéricos para la solución del modelo. Esto es viable desde dos perspectivas bien distintas: por una parte se propone que los estudiantes diseñen programas para 
resolver las ecuaciones o que utilicen para tal fin rutinas especializadas prediseñadas de los software especializados, como Octave o Matlab, que resuelven dichas ecuaciones (Quarteroni y Saleri, 2006).

Por otra parte, otra forma de validación de las soluciones encontradas es diseñando una experimentación (esto es, aventurando una formulación teórica que demuestre consistentemente la respuesta a una solución problemática), bien sea en el laboratorio con un montaje particular que dé cuenta de la situación propuesta o utilizando software para tal fin, como Quick Field, Algor u otro. Es necesario aquí tener en cuenta que se deben definir unos márgenes de error que serán los que definan la validez del modelo, que en caso de no ser satisfechos generan necesariamente una repetición del proceso de modelamiento para tratar de mejorar los resultados obtenidos, es decir, disminuir los márgenes de error cometidos.

Cuando la teoría es insuficiente para dar cuenta de un fenómeno o situación particular, el ingeniero experto necesita "crear" una nueva formulación teórica para explicar y resolver las situaciones que allí se le presenten (Chua, 1971); y en tanto se trata de un proceso de formación, se requiere que los estudiantes de ingeniería realicen esta labor con actividades diseñadas para tal fin. En suma, se espera que, como producto de la reflexión académica, sean capaces de construir o reconstruir una teoría matemática específica, reconociendo los elementos que allí intervienen, los axiomas, los teoremas, etc., es decir, los componentes específicos de toda teoría matemática.

\section{Modelamiento en el Aula de Clase}

En el marco del proyecto y en el curso de ecuaciones diferenciales ordinarias se buscaba que los estudiantes a través de la selección de una situación se aproximaran al ejercicio real de la ingeniería formulando modelos matemáticos (Kuně̌, 2012).

Así, algunos estudiantes interesados en comprender el comportamiento de la temperatura de un data center (un cuarto con servidores que deben permanecer en un rango de temperaturas determinadas con el objetivo de preservar su buen funcionamiento) determinan a partir del proceso las diversas variables que intervienen, lo que configura una situación compleja a la hora de plantear algún modelo matemático; sin embargo, para nuestro caso la situación se enmarcó dentro del campo de las ecuaciones diferenciales ordinarias de primer orden. En principio se plantea el problema más simple posible, se trata de establecer una relación funcional entre la temperatura del data center en función del tiempo, es decir, se propone que la variación de la temperatura del cuarto respecto al cambio en el tiempo es proporcional a la diferencia entre la temperatura del medio ambiente que lo rodea (TA) y la temperatura propia del cuarto (TC). De este modo los estudiantes han logrado establecer que el modelo matemático que permite predecir el comportamiento de la temperatura del cuarto obedece la ley de enfriamiento de Newton (Zill, 1997), ecuación (1). Los conceptos matemáticos se pueden aplicar a situaciones cotidianas para los ingenieros, como lo es el cambio en la temperatura del cuarto ¡donde trabajan permanentemente!

$$
\frac{d T(t)}{d t}=k\left(T_{C}-T_{A}\right)
$$

De esta forma, los estudiantes, luego de seleccionar una situación real y ubicarla en el campo, definen el sistema, las variables que intervienen, y consideran distintas formulaciones para el modelo correspondiente.

Otro grupo de estudiantes trabajó en una situación similar, pero en la cual querían determinar el comportamiento de la temperatura de un edificio en función del tiempo, teniendo en cuenta el calor generado por las personas, las luces y las máquinas dentro del edificio, situación que causa una razón de incremento en la temperatura, que se denominó $H(t)$, además, el calentamiento o enfriamiento proporcionado por la calefacción o el aire acondicionado, $U(t)$, y $(M(t))$ como el efecto de la 
temperatura exterior sobre el edificio. El modelo se presenta en la ecuación (2).

$$
\frac{d T(t)}{d t}=k[M(t)-T(t)]+H(t)+U(t)
$$

Las ecuaciones diferenciales fueron resueltas por los estudiantes, utilizando para este caso la técnica de la Transformada de Laplace. Así, los estudiantes no solo estudiaron en el curso de ecuaciones las formas de resolver ecuaciones, sino que aprendieron distintos planteamientos en los cuales las situaciones cotidianas o de ingeniería se podían escribir como ecuaciones diferenciales.

\section{Conclusiones}

Tras describir sintéticamente el proceso pedagógico propuesto, se mostró que los estudiantes (que han estado inmersos en la práctica pedagógica propuesta) apropian la lógica de aproximación teórica, basada en un proceso experimental que les exige emplear con rigor académico dominios conceptuales pertinentes (modelamiento matemático) y donde es preciso que se infieran posibilidades de solución fundamentadas en desarrollos particulares llevados a cabo por ellos mismos. Es claro, entonces, que la formación de ingenieros implica mayor acento en el componente especializado de la disciplina. No es lo mismo reconocer los procesos de solución de problemas tipo que verse obligado a formular problematizaciones situadas en contextos específicos, donde los problemas tipo solo sirven como puntos de referencia o de aproximación a la formulación de posibles soluciones.

La estrategia didáctica planteada, basada en el quehacer de los ingenieros, presenta desafíos para el docente, quien debe orientar, entre otras cosas, la selección de las situaciones que deberán ser modeladas, la técnica más apropiada al momento de resolver el modelo y el enfoque del análisis cuando los estudiantes han logrado resolver los modelos. Sin embargo, es preciso reconocer que, por cuestiones de tiempo, en general no es posible que en un curso de cuatro meses se logren contrastar los resultados teóricos con los resultados experimentales de la mayoría de los casos planteados.

\section{Referencias}

Bernstein B. y Díaz, M. (1985). Hacia una teoría del discurso pedagógico. Revista Colombiana de Educación, 15.

Chevallard, Y. (1998). La transposición didáctica. Del saber sabio al saber enseñado. Aique Grupo Editor.

Hritonenko N., Yatsenko Y. (2003). Applied mathematical modelling of engineering problems. Springer.

Kuneš K. (2012). similarity and modeling in science and engineering. Springer Link.

Kuhn, T. (2004). La estructura de las revoluciones científicas. México: Fondo de Cultura Económica.

Chua L. O. (1971). Memristor: the missing circuit element. IEEE Transactions on Circuit Theory, 18, 507-519.

Quarteroni, A. y Saleri, F. (2006). Cálculo científico con Matlab y Octave. Springer.

Richards, D. (2002). Advanced mathematical methods with Maple. Cambridge University Press.

Wolfram, S. y Beck, G. (1994). Mathematica: The student book. Addison Wesley Pub.

Zill, D. (1997). A first course in differential equations with modelling applications $\left(6^{\text {th }}\right.$ ed.). Brooks Cole Publishing Co. 